\title{
Lanthanide Contraction in Lanthanide Organic Frameworks: A Theoretical and Experimental Study
}

Gustavo Santana Silva ${ }^{\mathrm{a}}$, José Diogo L. Dutra ${ }^{\mathrm{a}}$, Nivan B. da Costa Jr. ${ }^{\mathrm{a}}$, Severino A. Junior ${ }^{\mathrm{b}}$ and Ricardo O. Freire ${ }^{\mathrm{a}, *}$

${ }^{a}$ Pople Computational Chemistry Laboratory, Department of Chemistry, Federal University of Sergipe-UFS, 49100-000 São Cristóvão, SE, Brazil ${ }^{b}$ Department of Elemental Chemistry, Federal University of Pernambuco - UFPE, 50590-470 Recife, PE, Brazil 


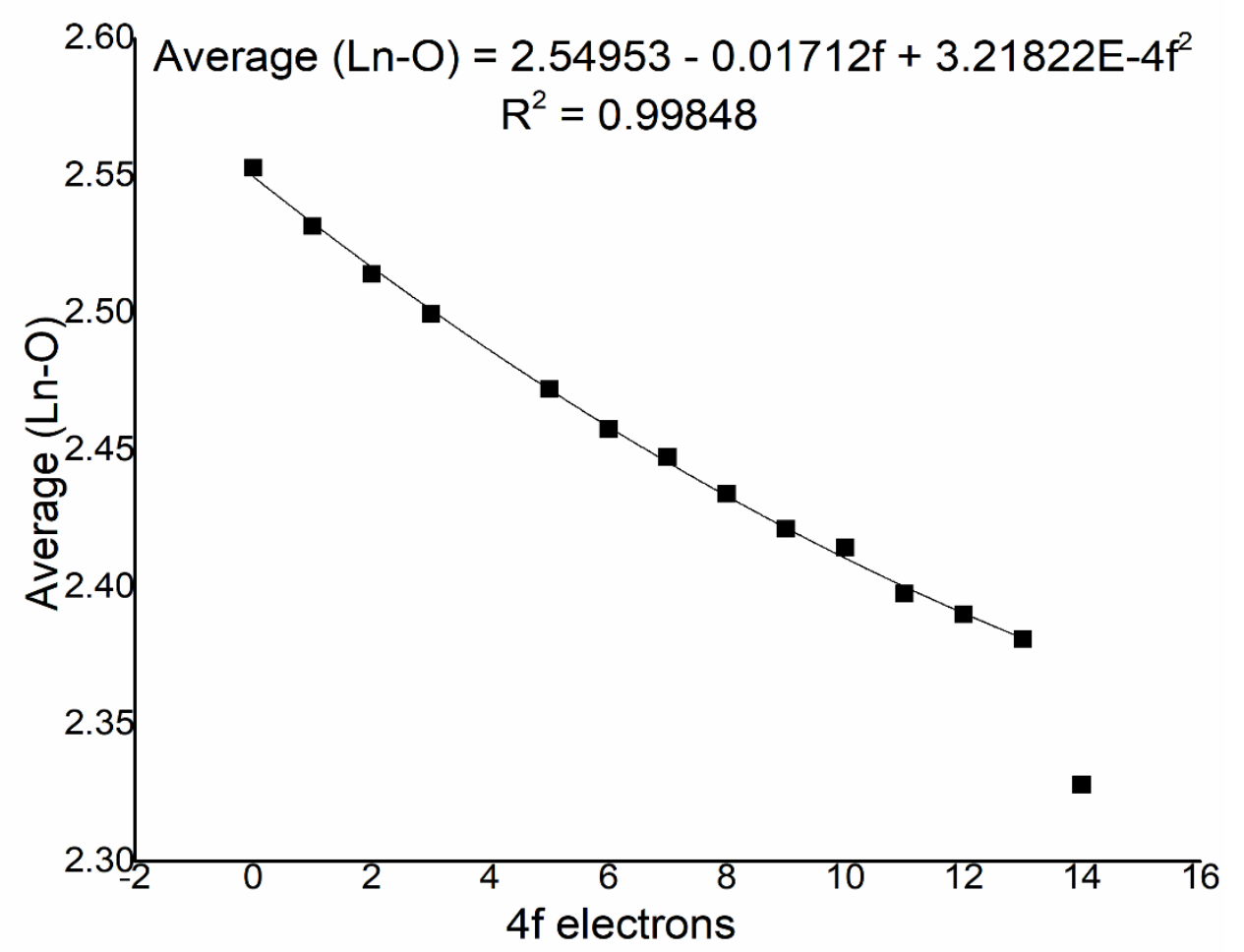

(A)

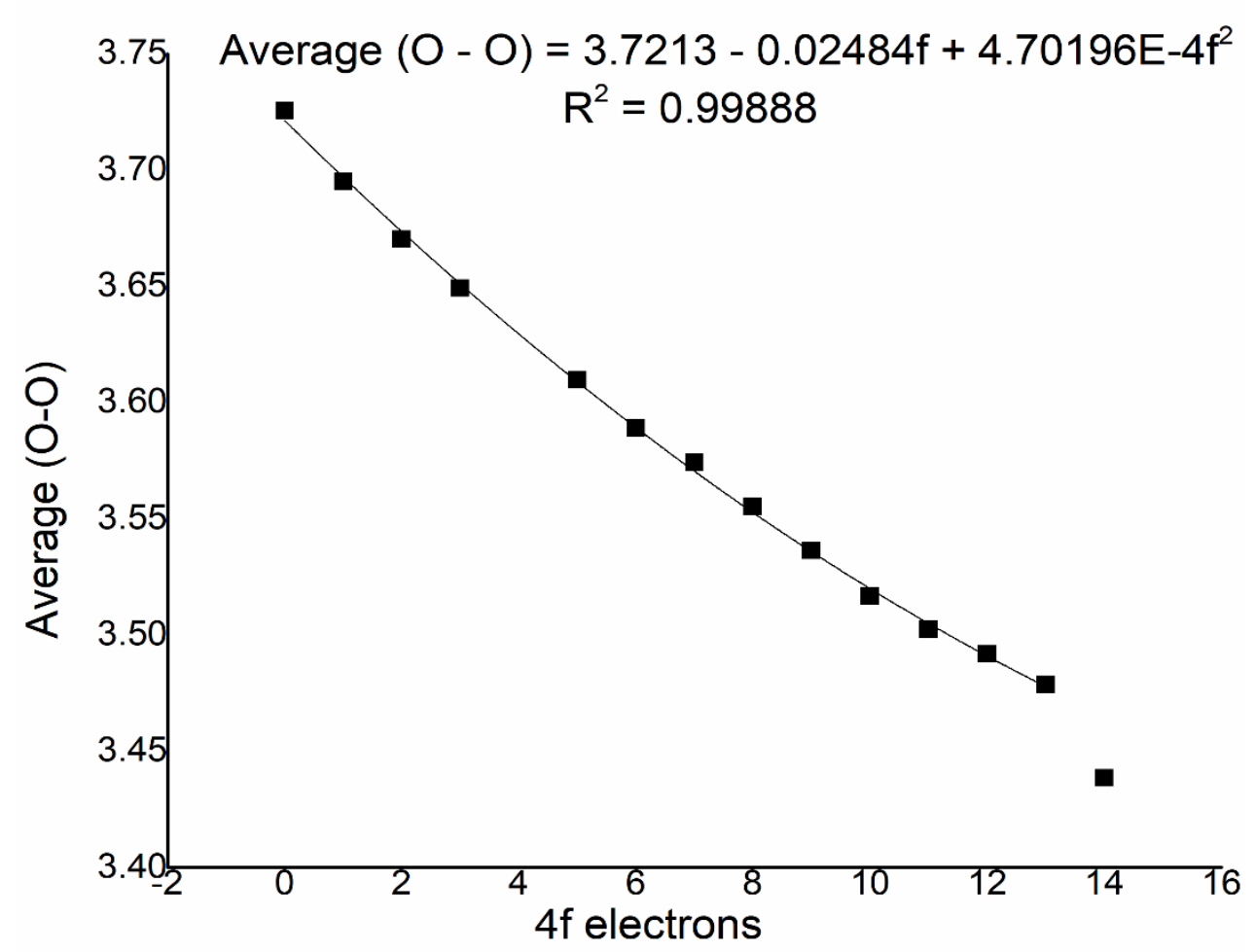

(B)

Figure S1. (A) Average of all Ln-O bond lengths and (B) of all O-O distances, for each $\mathrm{Ln}$, and the corresponding quadratic fit against the number of f-electrons, obtained for the full crystallographic geometries of the LOFs $\left[\mathrm{Ln}_{2}\left(\mathrm{C}_{4} \mathrm{H}_{4} \mathrm{O}_{4}\right)_{3}\left(\mathrm{H}_{2} \mathrm{O}\right)_{2}\right] . \mathrm{H}_{2} \mathrm{O}$. 


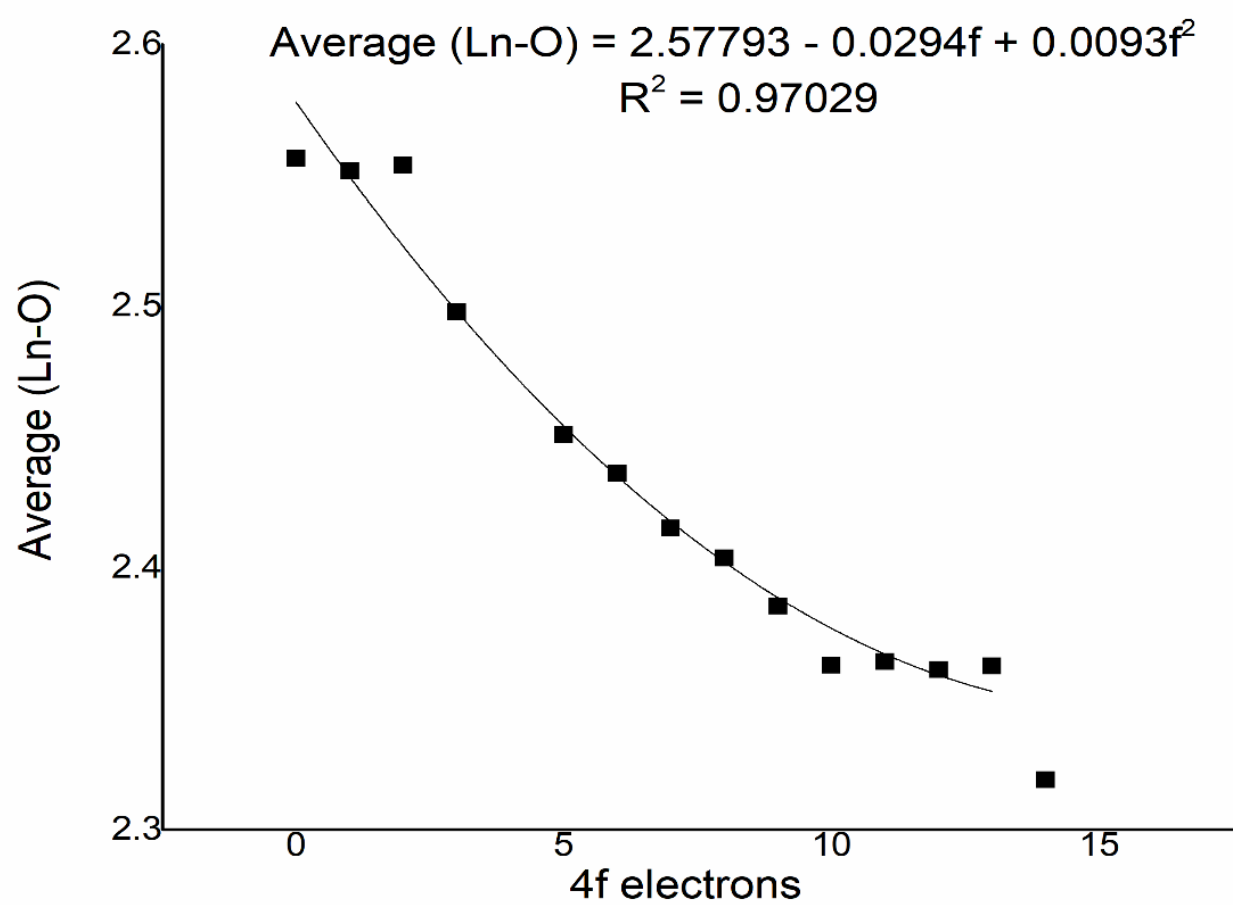

(A)

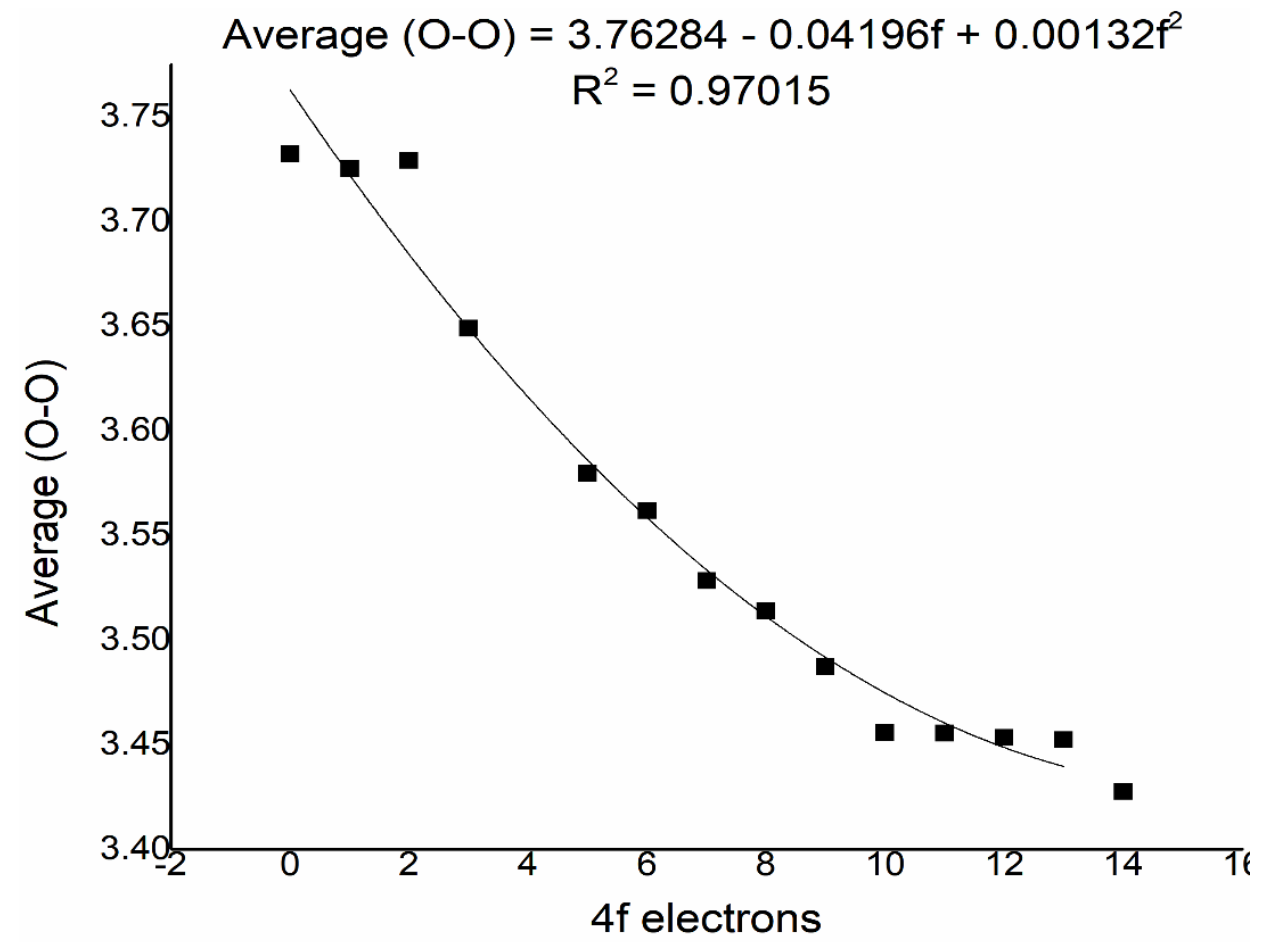

(B)

Figure S2. (A) Average of all Ln-O bond lengths and (B) of all O-O distances, for each Ln, and the corresponding quadratic fit against the number of f-electrons, obtained for the full Sparkle/PM3 geometries of the LOFs $\left[\mathrm{Ln}_{2}\left(\mathrm{C}_{4} \mathrm{H}_{4} \mathrm{O}_{4}\right)_{3}\left(\mathrm{H}_{2} \mathrm{O}\right)_{2}\right] \cdot \mathrm{H}_{2} \mathrm{O}$. 


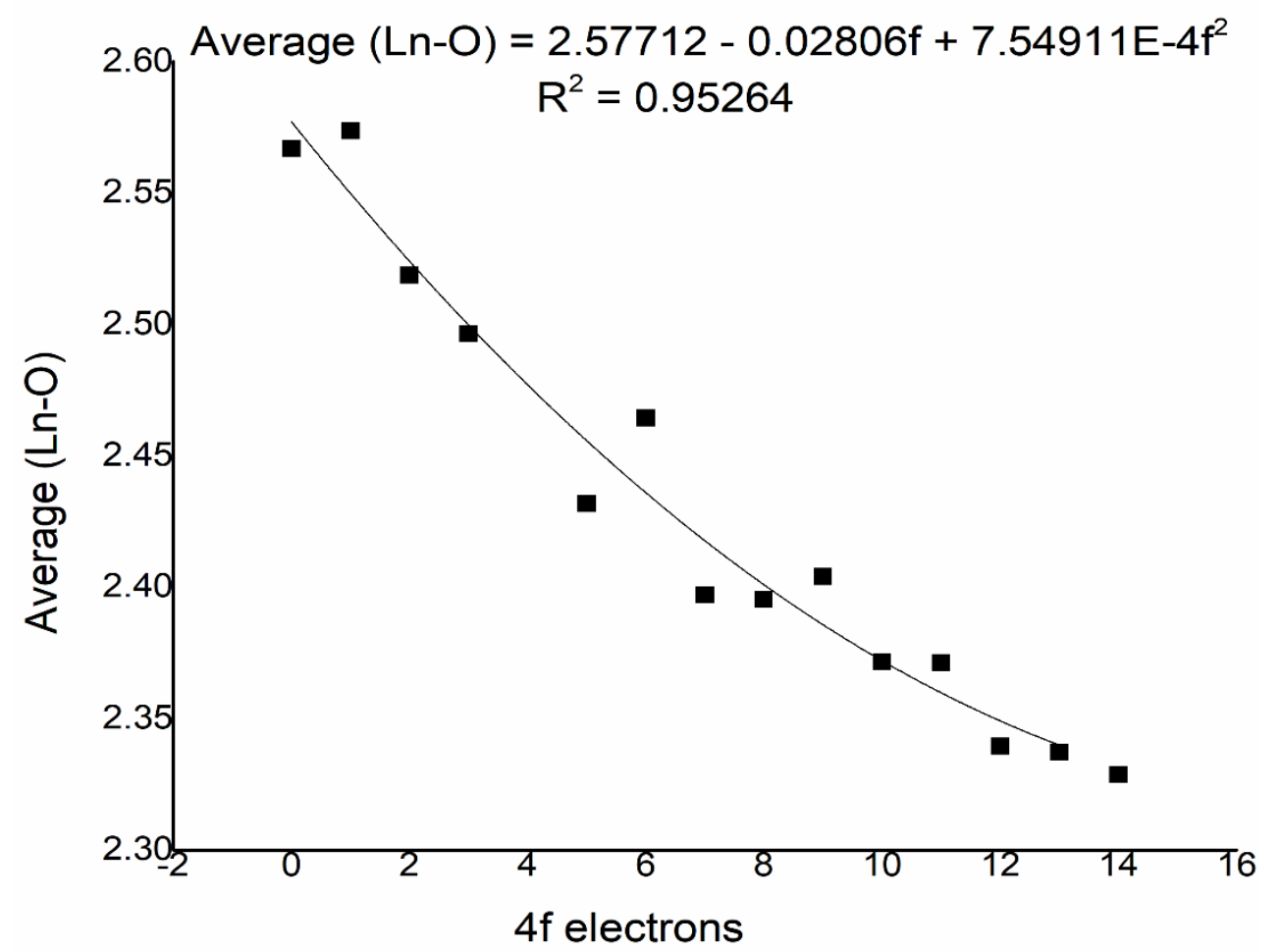

(A)

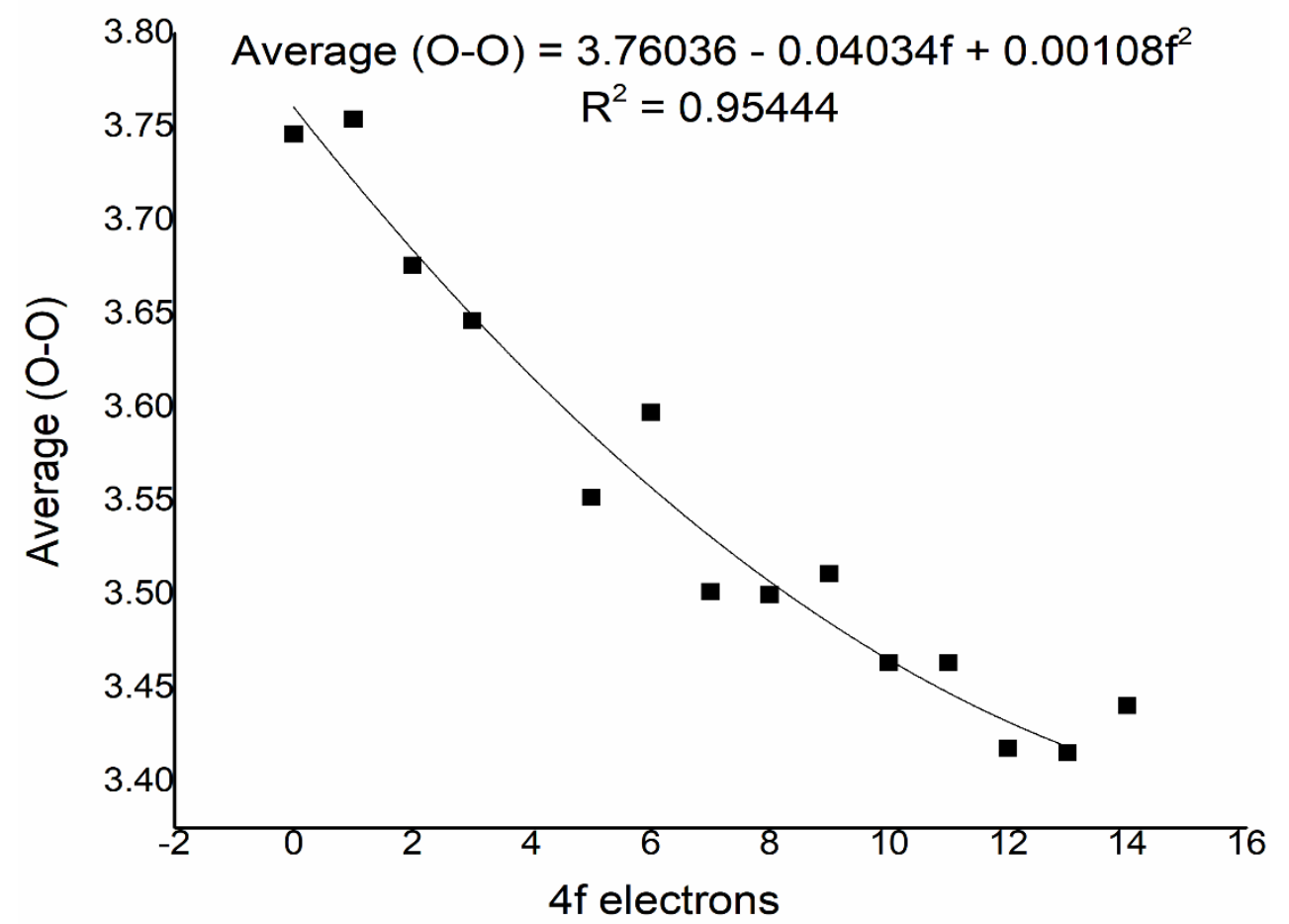

(B)

Figure S3. (A) Average of all $\mathrm{Ln}-\mathrm{O}$ bond lengths and (B) of all $\mathrm{O}-\mathrm{O}$ distances, for each $\mathrm{Ln}$, and the corresponding quadratic fit against the number of f-electrons, obtained for the full Sparkle/RM1 geometries of the LOFs $\left[\mathrm{Ln}_{2}\left(\mathrm{C}_{4} \mathrm{H}_{4} \mathrm{O}_{4}\right)_{3}\left(\mathrm{H}_{2} \mathrm{O}\right)_{2}\right] \cdot \mathrm{H}_{2} \mathrm{O}$. 


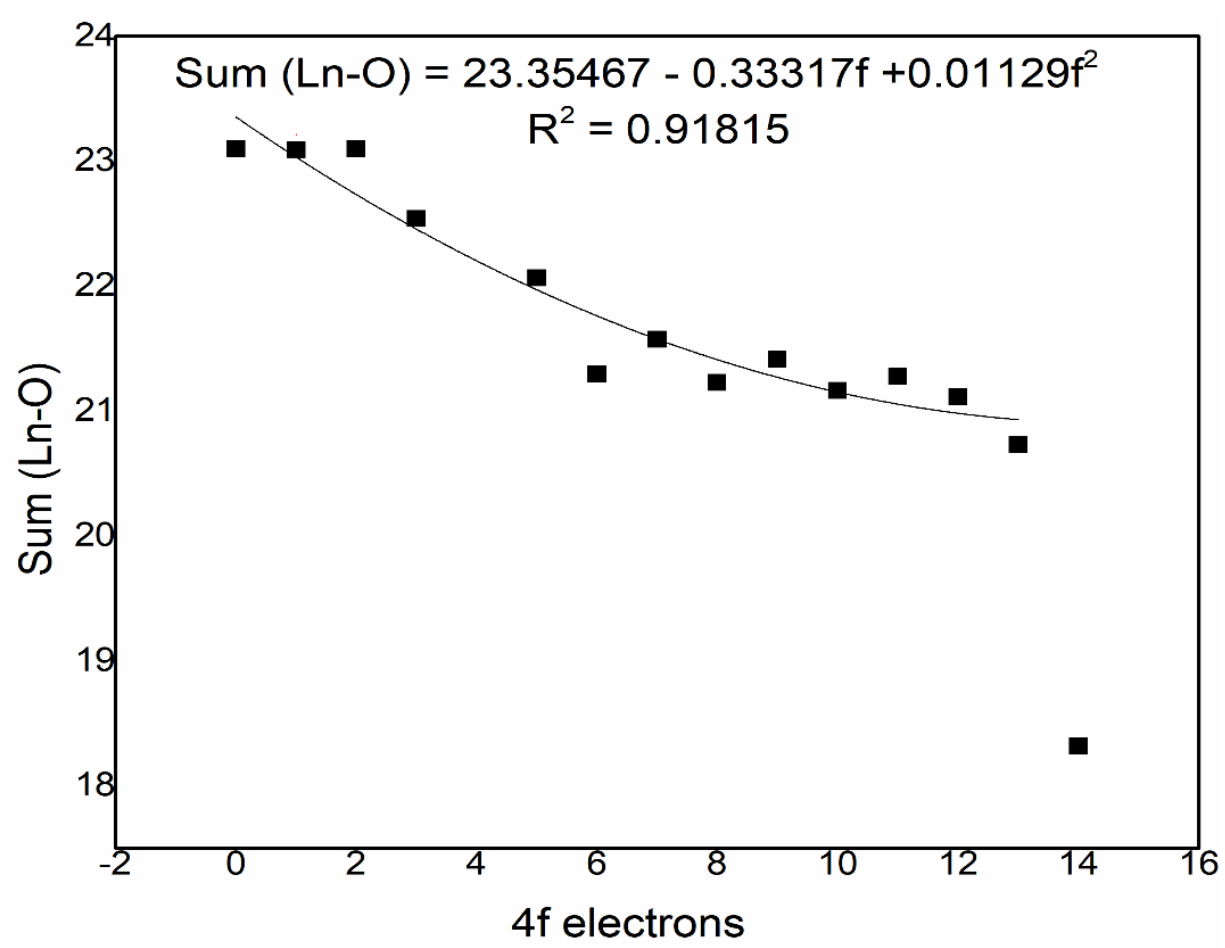

(A)

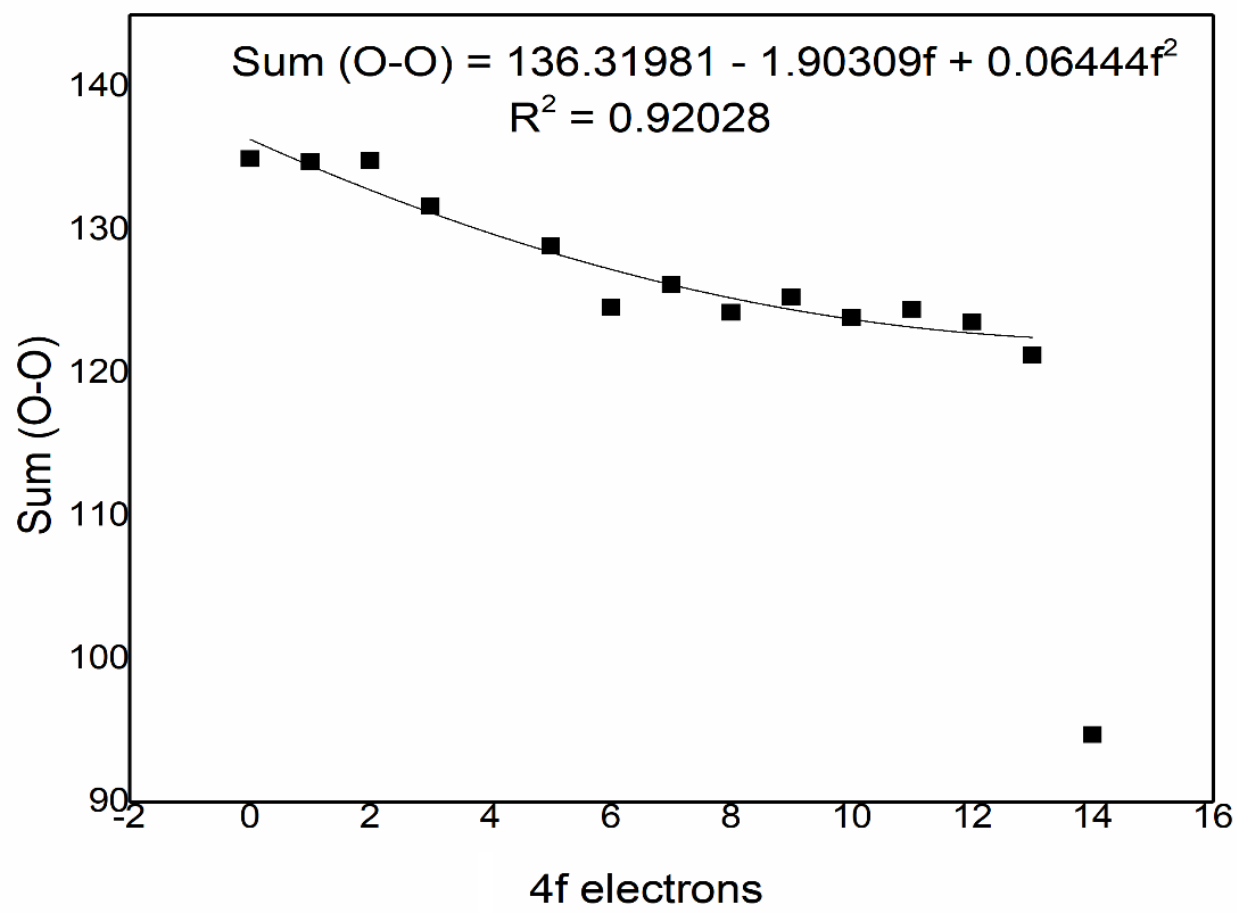

(B)

Figure S4. (A) Sum of all Ln-O bond lengths and (B) of all O-O distances, for each Ln, and the corresponding quadratic fit against the number of f-electrons, obtained for the full Sparkle/AM1 geometries of the LOFs $\left[\mathrm{Ln}_{2}\left(\mathrm{C}_{4} \mathrm{H}_{4} \mathrm{O}_{4}\right)_{3}\left(\mathrm{H}_{2} \mathrm{O}\right)_{2}\right] \cdot \mathrm{H}_{2} \mathrm{O}$. 


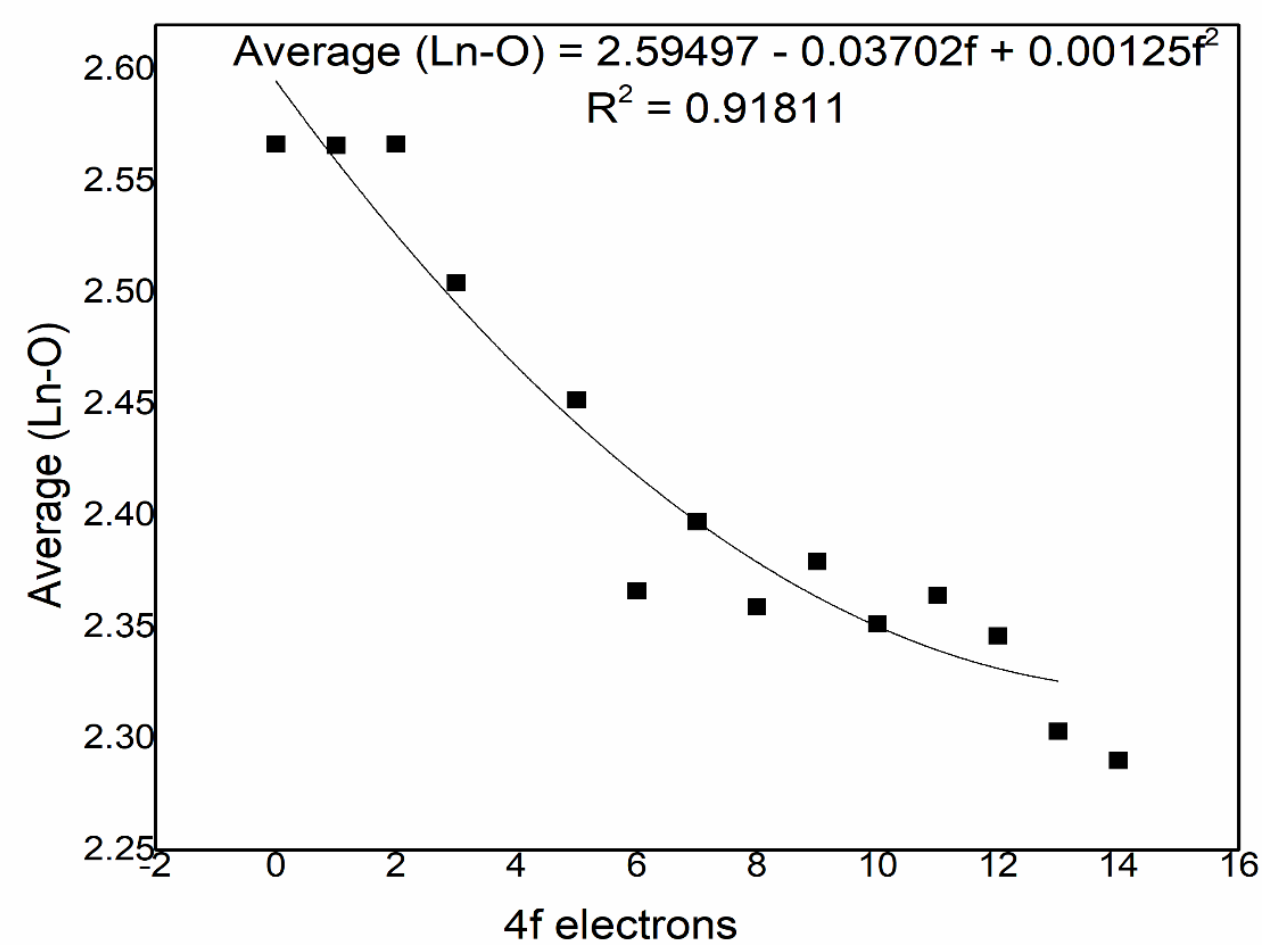

(A)

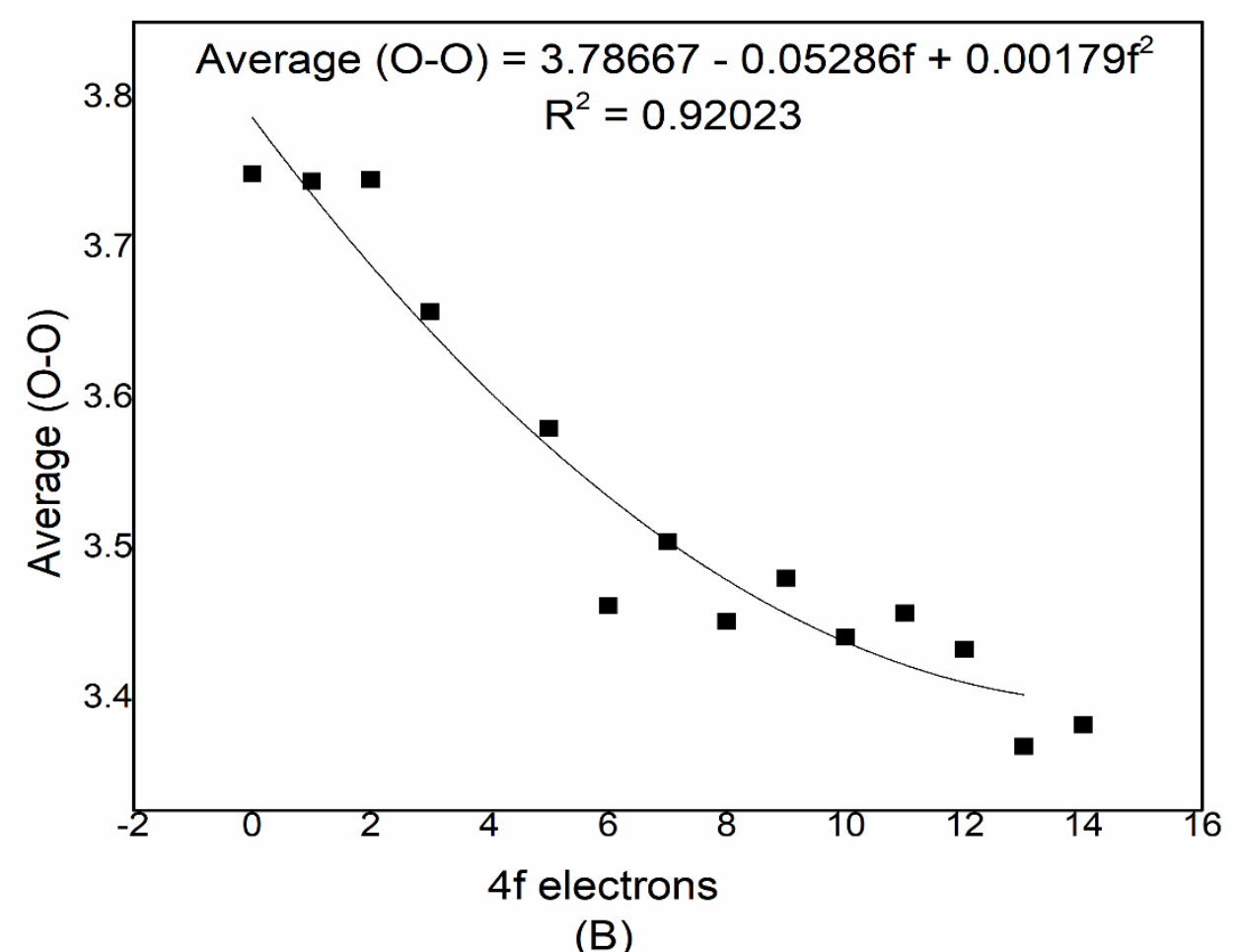

(B)

Figure S5. (A) Average of all Ln-O bond lengths and (B) of all O-O distances, for each Ln, and the corresponding quadratic fit against the number of f-electrons, obtained for the full Sparkle/AM1 geometries of the LOFs $\left[\mathrm{Ln}_{2}\left(\mathrm{C}_{4} \mathrm{H}_{4} \mathrm{O}_{4}\right)_{3}\left(\mathrm{H}_{2} \mathrm{O}\right)_{2}\right] . \mathrm{H}_{2} \mathrm{O}$. 

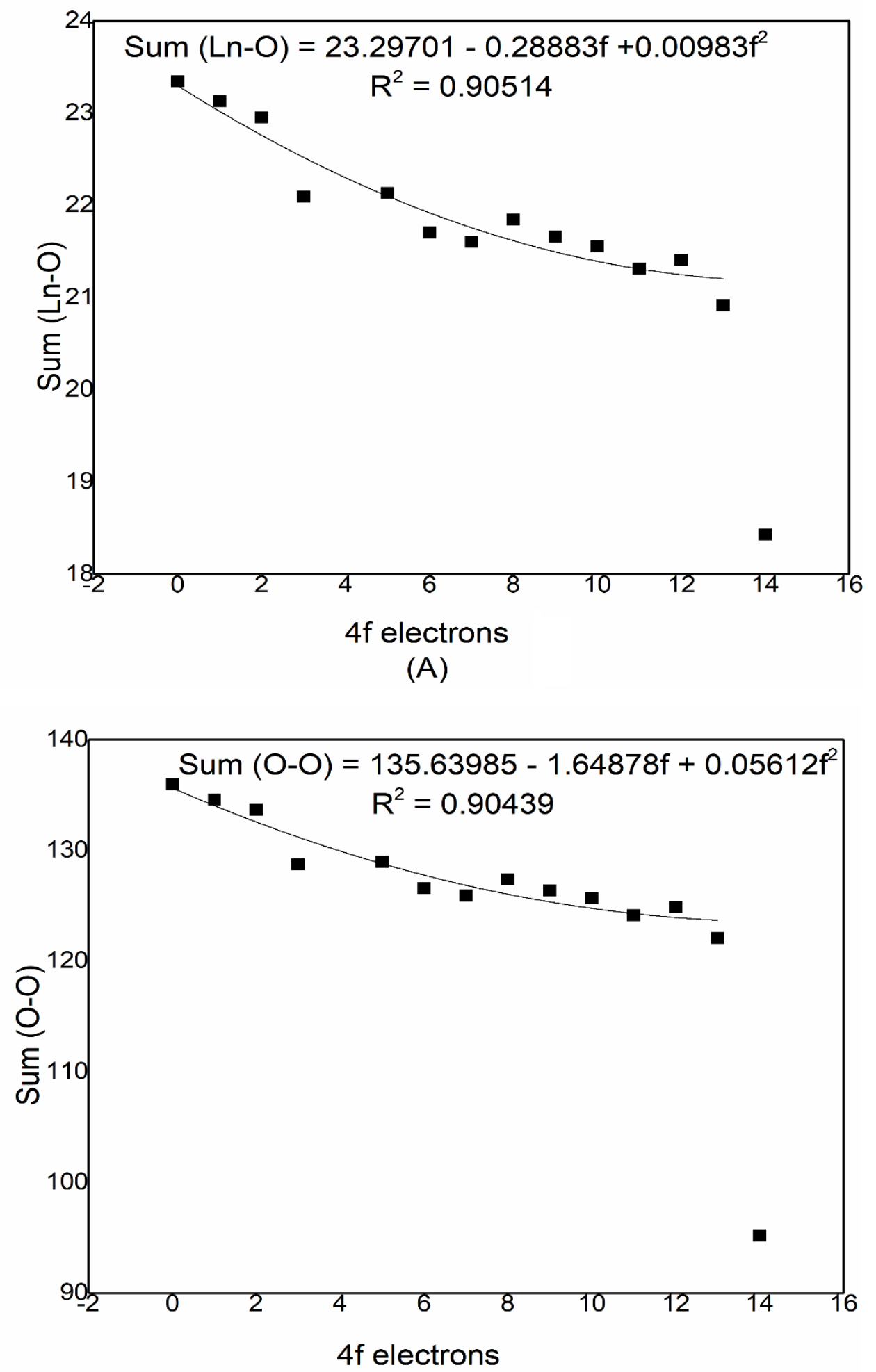

(B)

Figure S6. (A) Sum of all $\mathrm{Ln}-\mathrm{O}$ bond lengths and (B) of all O-O distances, for each $\mathrm{Ln}$, and the corresponding quadratic fit against the number of f-electrons, obtained for the full Sparkle/PM6 geometries of the LOFs $\left[\mathrm{Ln}_{2}\left(\mathrm{C}_{4} \mathrm{H}_{4} \mathrm{O}_{4}\right)_{3}\left(\mathrm{H}_{2} \mathrm{O}\right)_{2}\right] \cdot \mathrm{H}_{2} \mathrm{O}$. 


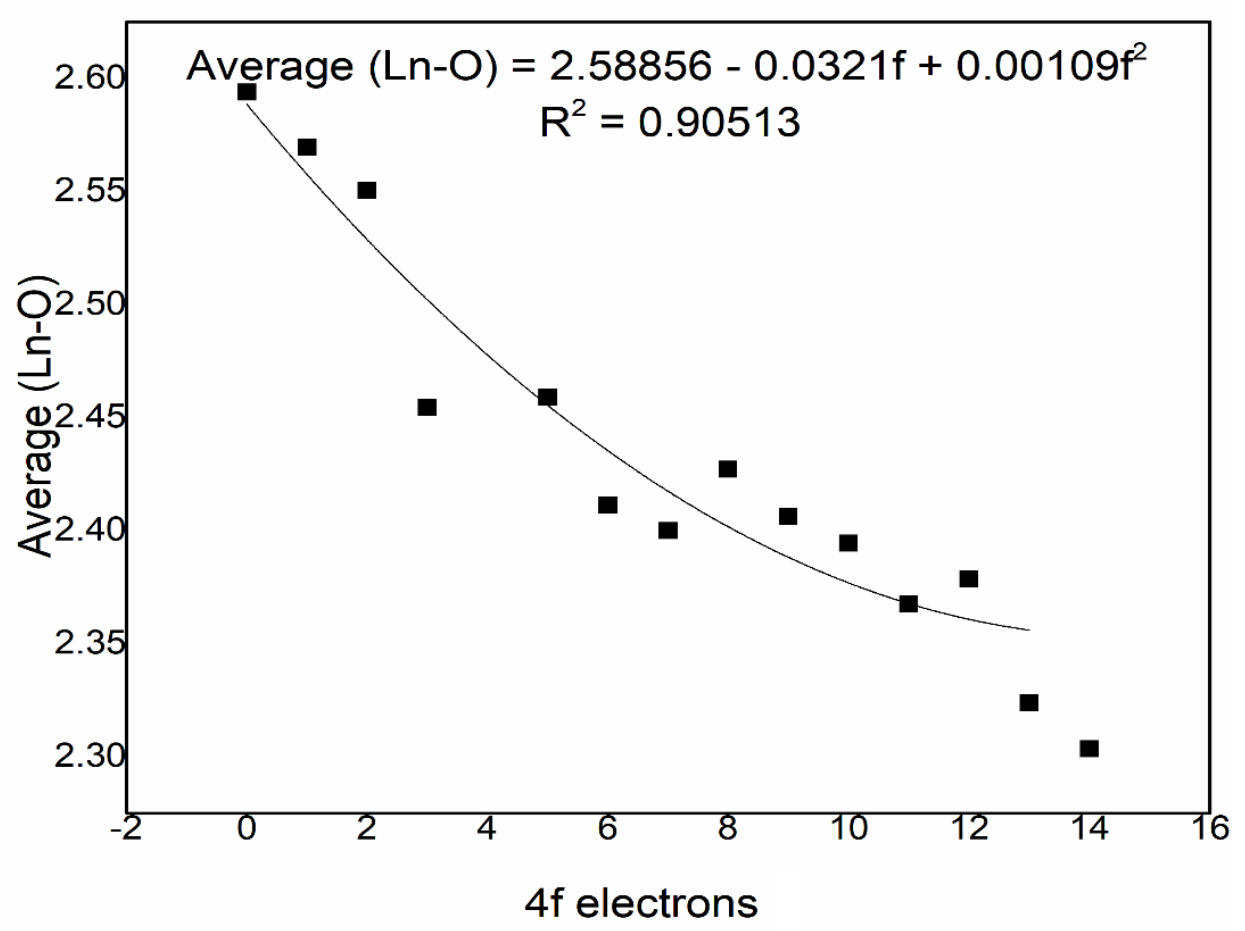

(A)

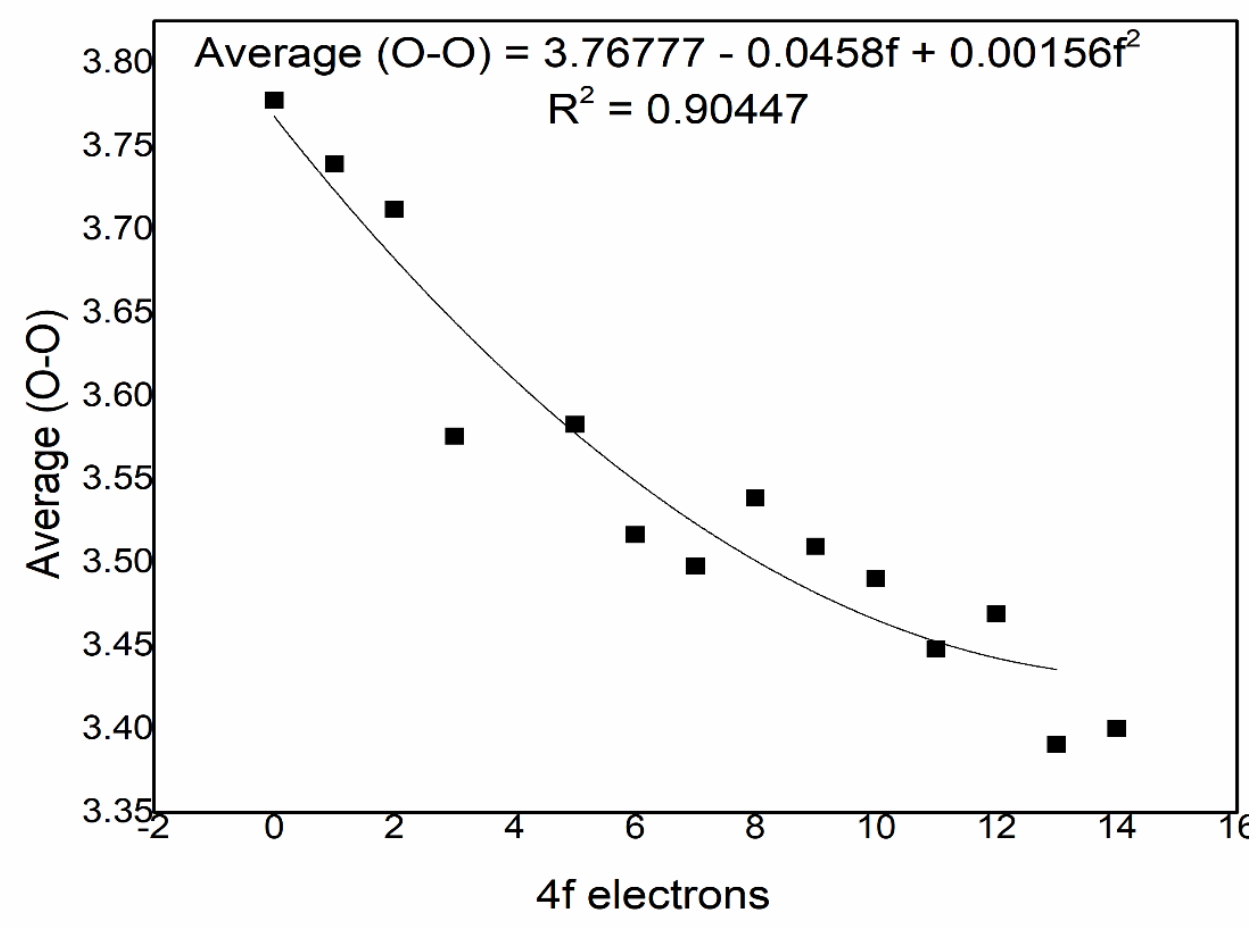

(B)

Figure S7. (A) Average of all Ln-O bond lengths and (B) of all O-O distances, for each Ln, and the corresponding quadratic fit against the number of f-electrons, obtained for the full Sparkle/PM6 geometries of the LOFs $\left[\mathrm{Ln}_{2}\left(\mathrm{C}_{4} \mathrm{H}_{4} \mathrm{O}_{4}\right)_{3}\left(\mathrm{H}_{2} \mathrm{O}\right)_{2}\right] \cdot \mathrm{H}_{2} \mathrm{O}$. 


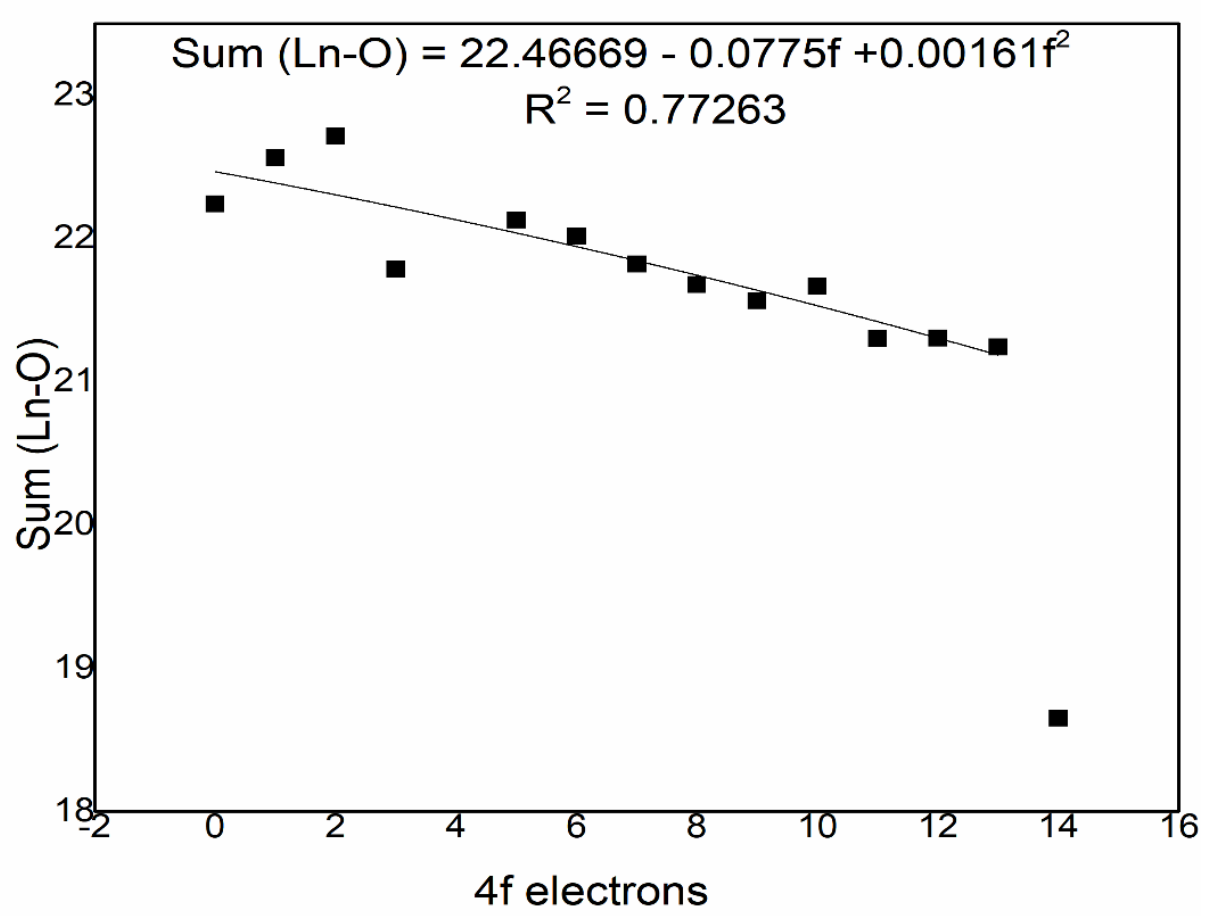

(A)

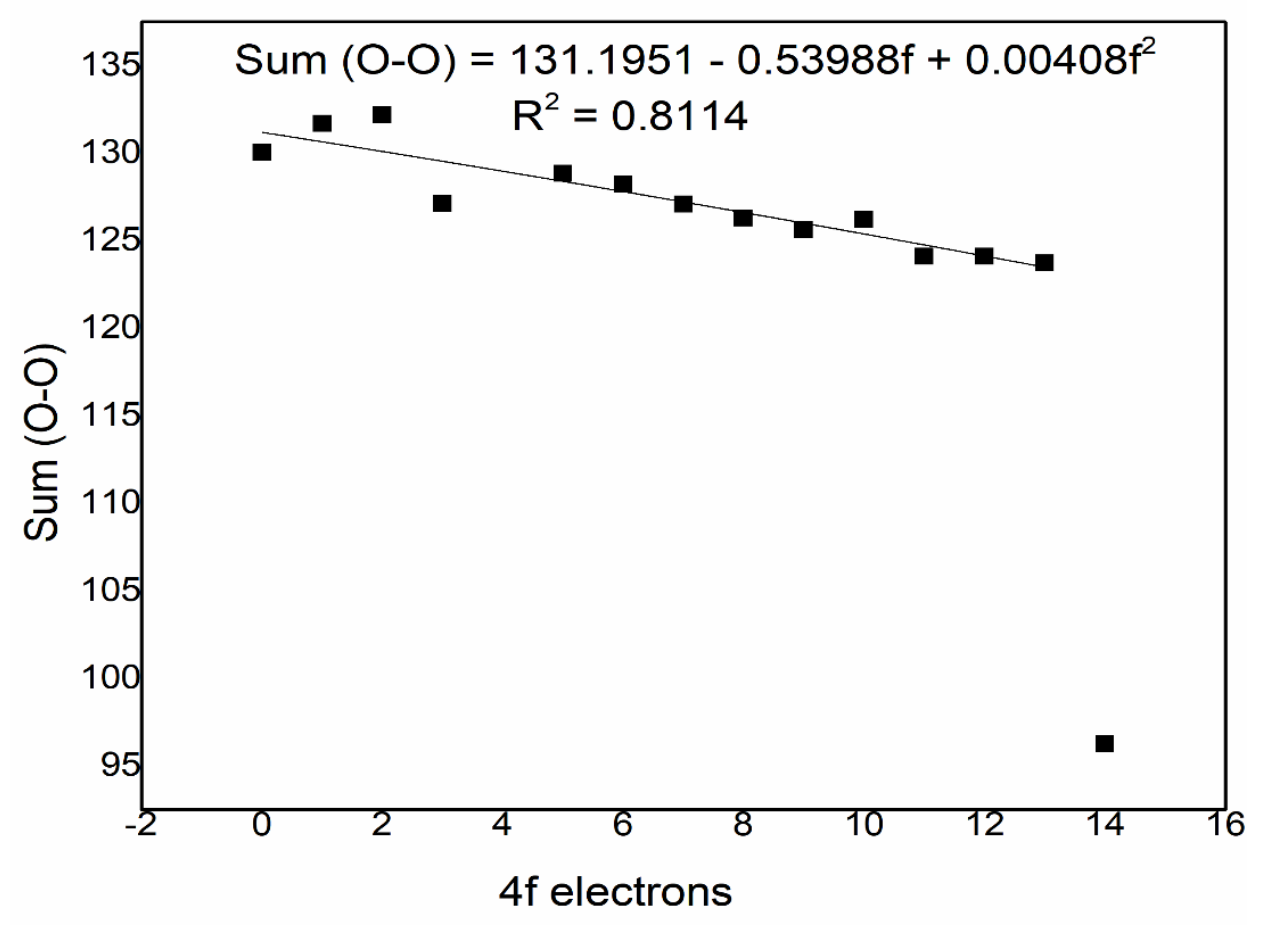

(B)

Figure S8. (A) Sum of all $\mathrm{Ln}-\mathrm{O}$ bond lengths and (B) of all O-O distances, for each $\mathrm{Ln}$, and the corresponding quadratic fit against the number of f-electrons, obtained for the full Sparkle/PM7 geometries of the LOFs $\left[\mathrm{Ln}_{2}\left(\mathrm{C}_{4} \mathrm{H}_{4} \mathrm{O}_{4}\right)_{3}\left(\mathrm{H}_{2} \mathrm{O}\right)_{2}\right] \cdot \mathrm{H}_{2} \mathrm{O}$. 


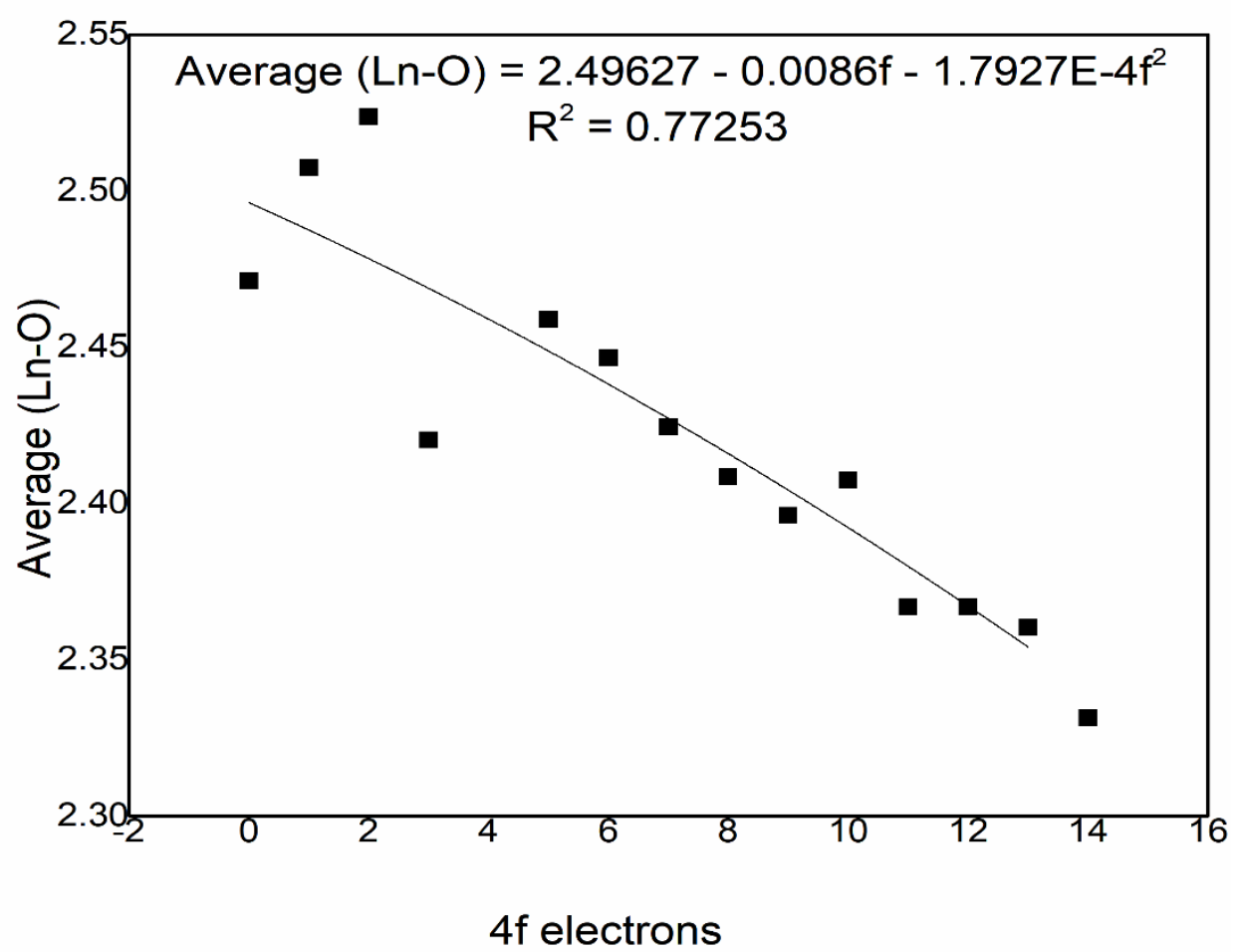

(A)

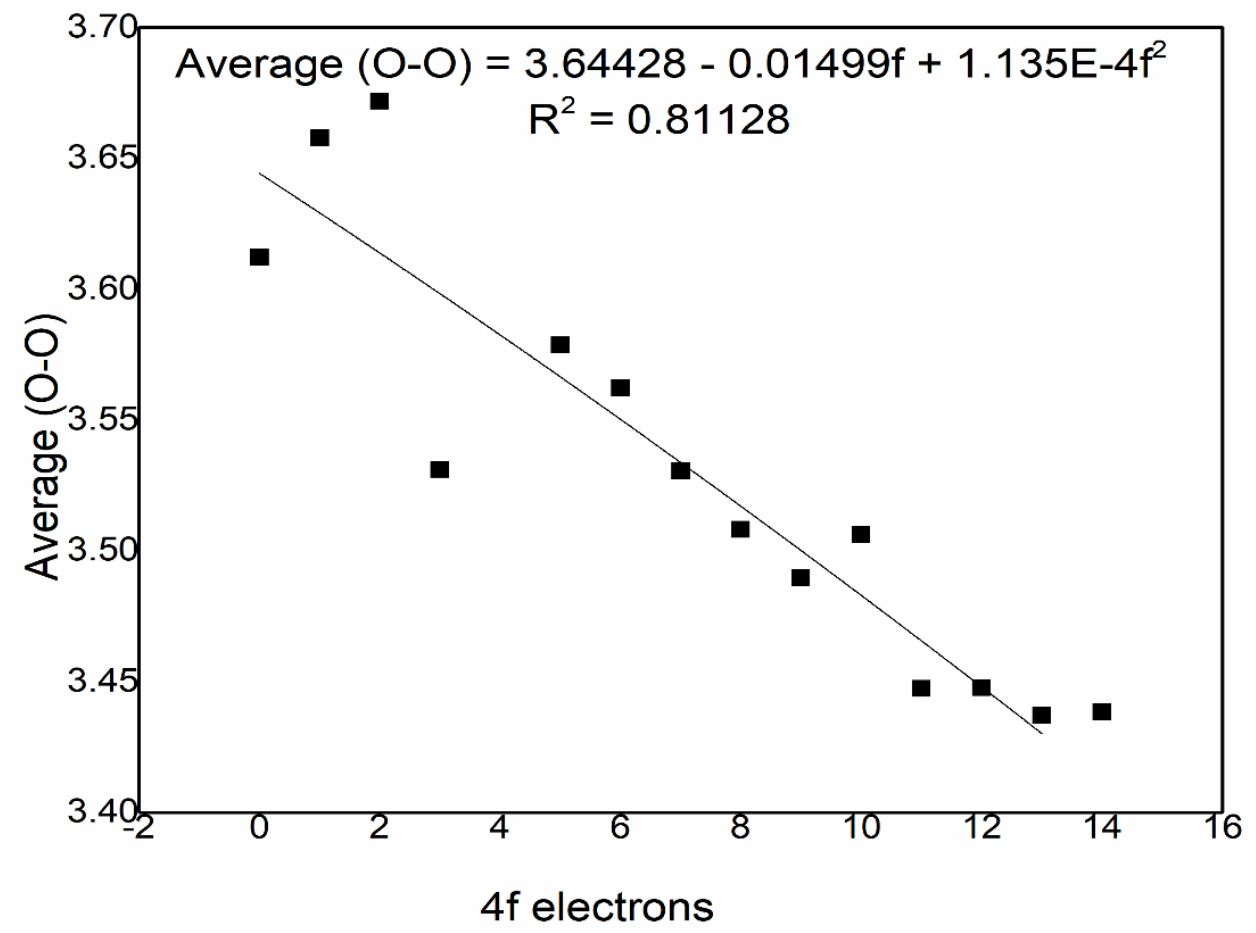

(B)

Figure S9. (A) Average of all Ln-O bond lengths and (B) of all O-O distances, for each $\mathrm{Ln}$, and the corresponding quadratic fit against the number of f-electrons, obtained for the full Sparkle/PM7 geometries of the LOFs $\left[\mathrm{Ln}_{2}\left(\mathrm{C}_{4} \mathrm{H}_{4} \mathrm{O}_{4}\right)_{3}\left(\mathrm{H}_{2} \mathrm{O}\right)_{2}\right] \cdot \mathrm{H}_{2} \mathrm{O}$. 


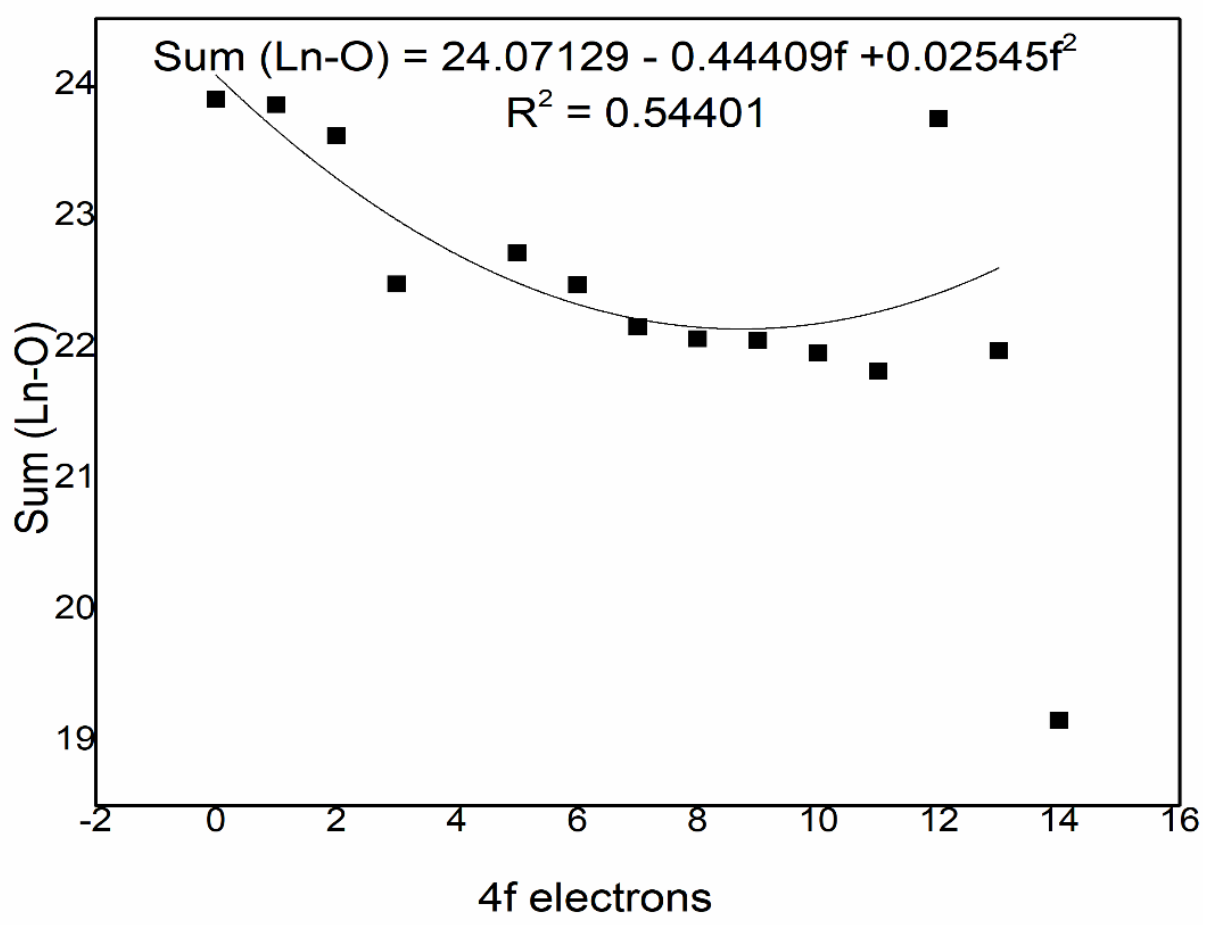

(A)

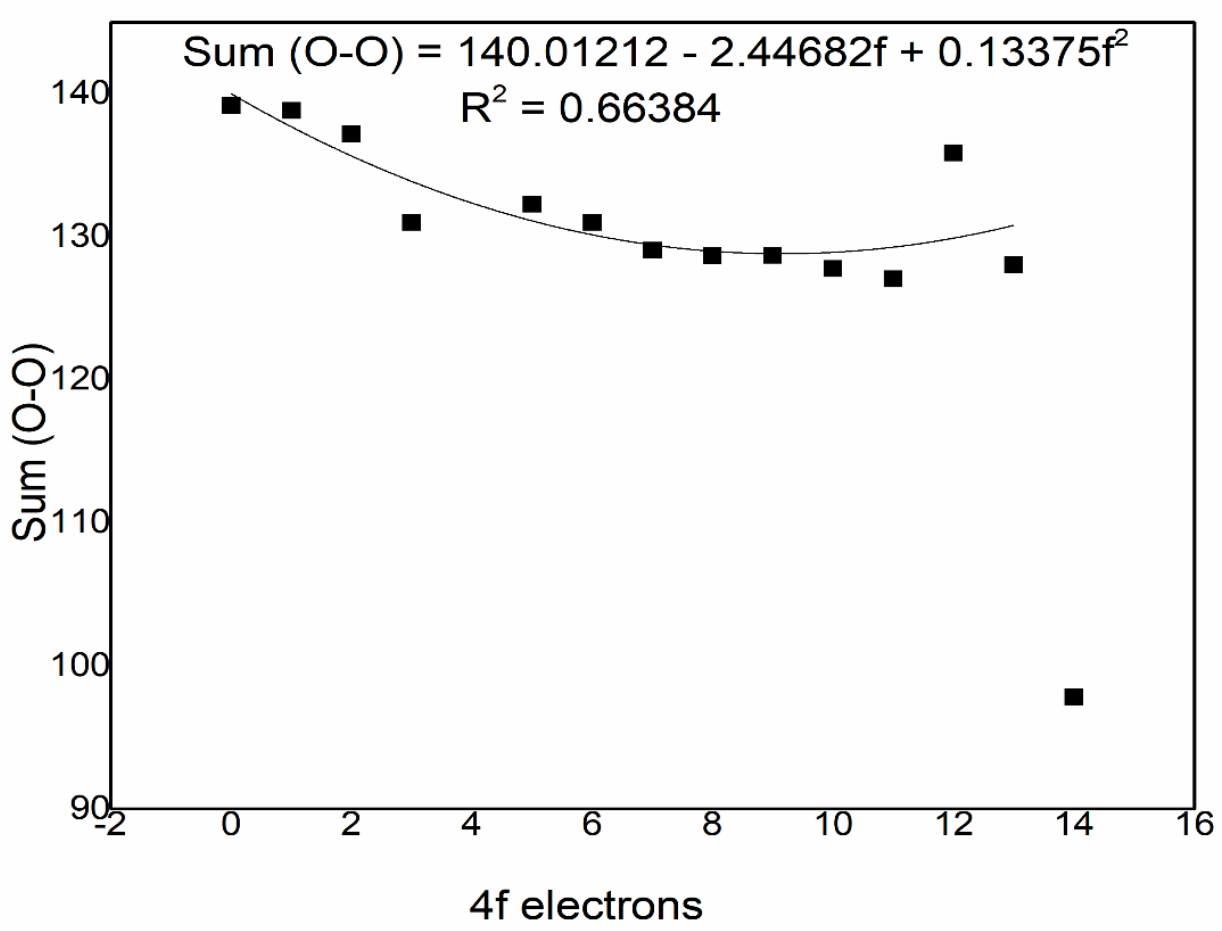

(B)

Figure S10. (A) Sum of all Ln-O bond lengths and (B) of all O-O distances, for each $\mathrm{Ln}$, and the corresponding quadratic fit against the number of f-electrons, obtained for the full RM1 geometries of the LOFs $\left[\mathrm{Ln}_{2}\left(\mathrm{C}_{4} \mathrm{H}_{4} \mathrm{O}_{4}\right)_{3}\left(\mathrm{H}_{2} \mathrm{O}\right)_{2}\right] \cdot \mathrm{H}_{2} \mathrm{O}$. 


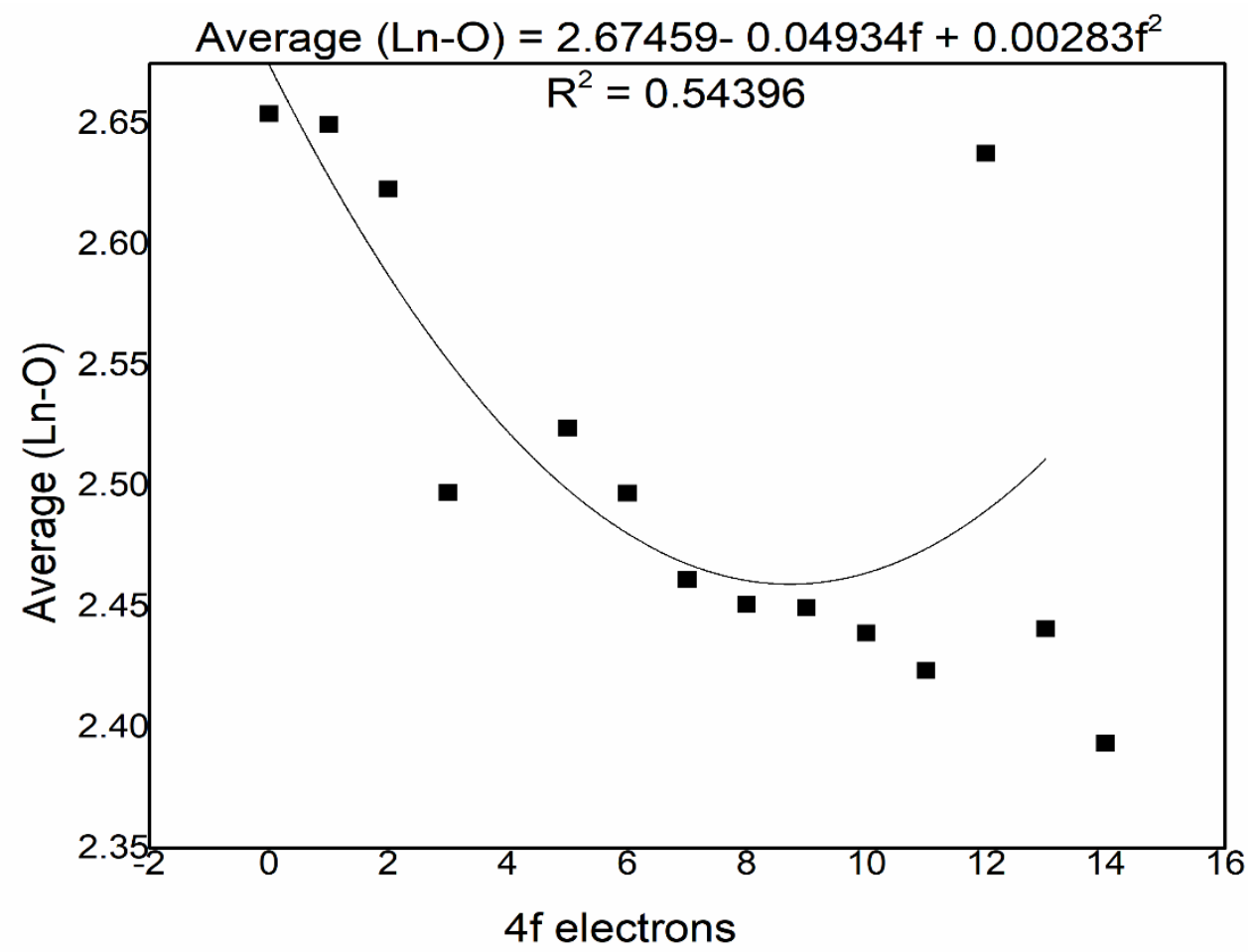

(A)

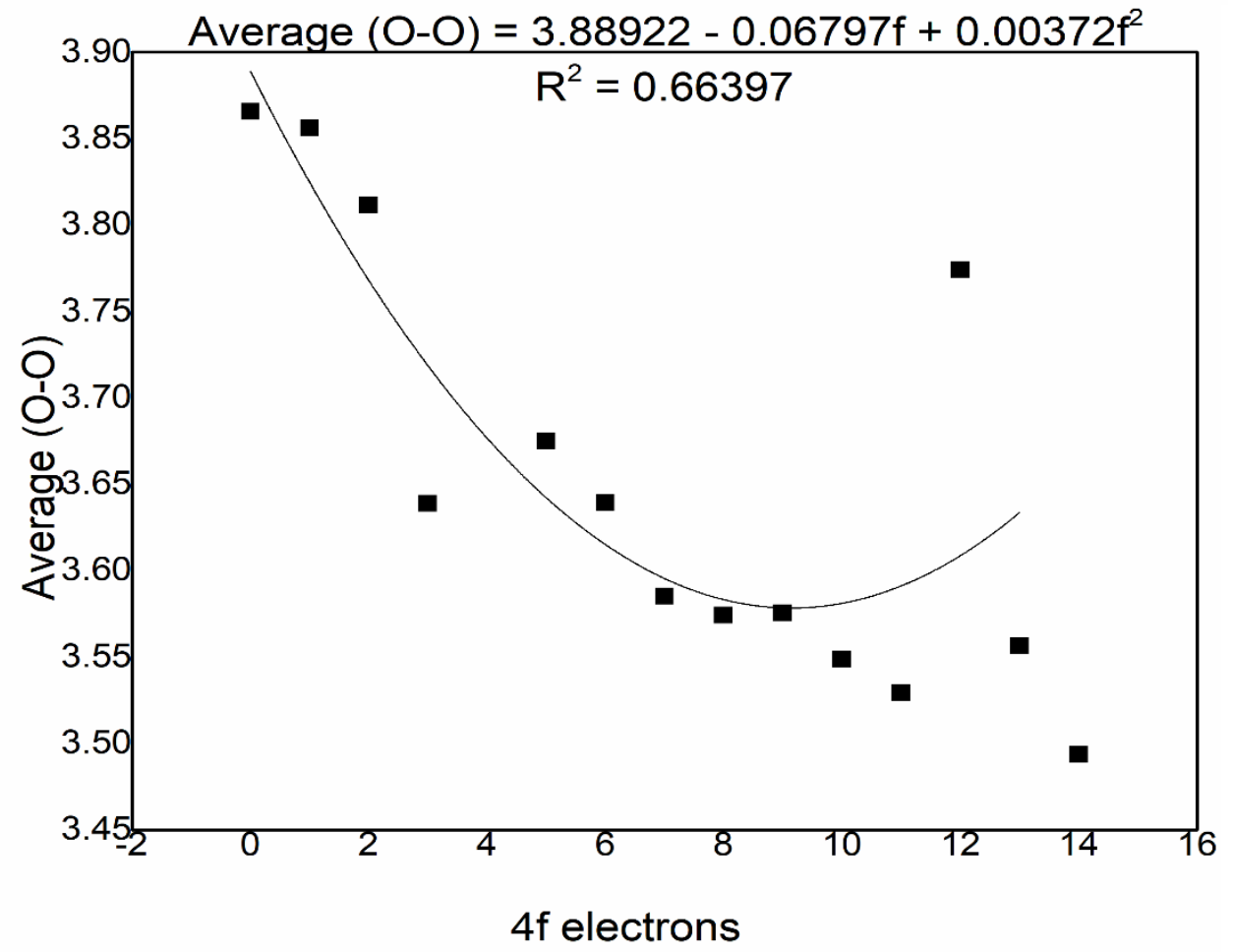

(B)

Figure S11. (A) Average of all $\mathrm{Ln}-\mathrm{O}$ bond lengths and (B) of all $\mathrm{O}-\mathrm{O}$ distances, for each $\mathrm{Ln}$, and the corresponding quadratic fit against the number of f-electrons, obtained for the full RM1 geometries of the LOFs $\left[\mathrm{Ln}_{2}\left(\mathrm{C}_{4} \mathrm{H}_{4} \mathrm{O}_{4}\right)_{3}\left(\mathrm{H}_{2} \mathrm{O}\right)_{2}\right] . \mathrm{H}_{2} \mathrm{O}$. 
Table S1. Sum of all Ln-O bond lengths, $\Sigma_{\mathrm{Ln}-\mathrm{O}}$, and sum of all $\mathrm{O}-\mathrm{O}$ distances, $\Sigma_{\mathrm{O}-\mathrm{O}}$, obtained from Sparkle/AM1 full geometry optimizations of the LOFs $\left[\mathrm{Ln}_{2}\left(\mathrm{C}_{4} \mathrm{H}_{4} \mathrm{O}_{4}\right)_{3}\left(\mathrm{H}_{2} \mathrm{O}\right)_{2}\right] \cdot \mathrm{H}_{2} \mathrm{O}$, where $\mathrm{Ln}$ is $\mathrm{La}-\mathrm{Lu}$ (except Pm).

\begin{tabular}{ccccc}
\hline f electrons $(\mathrm{Ln})$ & $\Sigma_{\text {Ln-O }}(\AA)$ & Average $(\AA)$ & $\Sigma_{\text {O-O }}(\AA)$ & Average $(\AA)$ \\
\hline $0(\mathrm{La})$ & 23.0991 & 2.5666 & 134.9512 & 3.7486 \\
$1(\mathrm{Ce})$ & 23.0933 & 2.5659 & 134.7791 & 3.7439 \\
$2(\mathrm{Pr})$ & 23.0988 & 2.5665 & 134.8195 & 3.7450 \\
$3(\mathrm{Nd})$ & 22.4806 & 2.5044 & 131.6541 & 3.6571 \\
$4(\mathrm{Pm})$ & -- & --- & -- & -- \\
$5(\mathrm{Sm})$ & 22.5392 & 2.4518 & 128.8571 & 3.5794 \\
$6(\mathrm{Eu})$ & 22.0660 & 2.3662 & 124.5932 & 3.4609 \\
$7(\mathrm{Gd})$ & 21.2960 & 2.3972 & 126.1357 & 3.5038 \\
$8(\mathrm{~Tb})$ & 21.5749 & 2.3589 & 124.2190 & 3.4505 \\
$9(\mathrm{Dy})$ & 21.2301 & 2.3792 & 125.2634 & 3.4795 \\
$10(\mathrm{Ho})$ & 21.4125 & 2.3514 & 123.8493 & 3.4403 \\
$11(\mathrm{Er})$ & 21.1630 & 2.3642 & 124.4243 & 3.4562 \\
$12(\mathrm{Tm})$ & 21.2776 & 2.3459 & 123.5578 & 3.4322 \\
$13(\mathrm{Yb})$ & 21.1127 & 2.3032 & 121.2302 & 3.3675 \\
$14(\mathrm{Lu})$ & 20.7291 & 2.2900 & 94.6863 & 3.3817 \\
$\mathrm{~d}_{\mathrm{La}} / \mathrm{d}_{\mathrm{Lu}}$ & 1.1143 & 1.1208 & 1.4252 & 1.1085 \\
\hline
\end{tabular}

Table S2. Sum of all Ln-O bond lengths, $\Sigma_{\mathrm{Ln}-\mathrm{O}}$, and sum of all $\mathrm{O}-\mathrm{O}$ distances, $\Sigma_{\mathrm{O}-\mathrm{O}}$, obtained from Sparkle/PM6 full geometry optimizations of the LOFs $\left[\mathrm{Ln}_{2}\left(\mathrm{C}_{4} \mathrm{H}_{4} \mathrm{O}_{4}\right)_{3}\left(\mathrm{H}_{2} \mathrm{O}\right)_{2}\right] . \mathrm{H}_{2} \mathrm{O}$, where $\mathrm{Ln}$ is $\mathrm{La}-\mathrm{Lu}$ (except Pm).

\begin{tabular}{ccccc}
\hline f electrons $(\mathrm{Ln})$ & $\Sigma_{\text {Ln-O }}(\AA)$ & Average $(\AA)$ & $\Sigma_{\text {O-O }}(\AA)$ & Average $(\AA)$ \\
\hline $0(\mathrm{La})$ & 23.3453 & 2.5939 & 135.9830 & 3.7773 \\
$1(\mathrm{Ce})$ & 23.1247 & 2.5694 & 134.6077 & 3.7391 \\
$2(\mathrm{Pr})$ & 22.9544 & 2.5505 & 133.6372 & 3.7121 \\
$3(\mathrm{Nd})$ & 22.0893 & 2.4544 & 128.7258 & 3.5757 \\
$4(\mathrm{Pm})$ & -- & --- & -- & -- \\
$5(\mathrm{Sm})$ & 22.1310 & 2.4590 & 128.9820 & 3.5828 \\
$6(\mathrm{Eu})$ & 21.7021 & 2.4113 & 126.5966 & 3.5166 \\
$7(\mathrm{Gd})$ & 21.6003 & 2.4000 & 125.9178 & 3.4977 \\
$8(\mathrm{~Tb})$ & 21.8441 & 2.4271 & 127.3948 & 3.5387 \\
$9(\mathrm{Dy})$ & 21.6577 & 2.4064 & 126.3508 & 3.5097 \\
$10(\mathrm{Ho})$ & 21.5502 & 2.3945 & 125.6509 & 3.4903 \\
$11(\mathrm{Er})$ & 21.3080 & 2.3676 & 124.1347 & 3.4482 \\
$12(\mathrm{Tm})$ & 21.4062 & 2.3785 & 124.8891 & 3.4691 \\
$13(\mathrm{Yb})$ & 20.9149 & 2.3239 & 122.0679 & 3.3908 \\
$14(\mathrm{Lu})$ & 18.4274 & 2.3034 & 95.2049 & 3.4002 \\
$\mathrm{~d}_{\mathrm{La}} / \mathrm{d}_{\mathrm{Lu}}$ & 1.2669 & 1.1261 & 1.4283 & 1.1109 \\
\hline
\end{tabular}


Table S3. Sum of all Ln-O bond lengths, $\Sigma_{\mathrm{Ln}-\mathrm{O}}$, and sum of all $\mathrm{O}-\mathrm{O}$ distances, $\Sigma_{\mathrm{O}-\mathrm{O}}$, obtained from Sparkle/PM7 full geometry optimizations of the LOFs $\left[\mathrm{Ln}_{2}\left(\mathrm{C}_{4} \mathrm{H}_{4} \mathrm{O}_{4}\right)_{3}\left(\mathrm{H}_{2} \mathrm{O}\right)_{2}\right] \cdot \mathrm{H}_{2} \mathrm{O}$, where $\mathrm{Ln}$ is $\mathrm{La}-\mathrm{Lu}$ (except Pm).

\begin{tabular}{ccccc}
\hline f electrons $(\mathrm{Ln})$ & $\Sigma_{\text {Ln-O }}(\AA)$ & Average $(\AA)$ & $\Sigma_{\text {O-O }}(\AA)$ & Average $(\AA)$ \\
\hline $0(\mathrm{La})$ & 22.6786 & 2.5198 & 132.3885 & 3.6775 \\
$1(\mathrm{Ce})$ & 22.5710 & 2.5079 & 131.7182 & 3.6588 \\
$2(\mathrm{Pr})$ & 22.7273 & 2.5253 & 132.3579 & 3.6766 \\
$3(\mathrm{Nd})$ & 22.0331 & 2.4481 & 128.2869 & 3.5635 \\
$4(\mathrm{Pm})$ & -- & --- & -- & -- \\
$5(\mathrm{Sm})$ & 22.1309 & 2.4590 & 128.8248 & 3.5785 \\
$6(\mathrm{Eu})$ & 22.0175 & 2.4464 & 128.2452 & 3.5624 \\
$7(\mathrm{Gd})$ & 21.8184 & 2.4243 & 127.1006 & 3.5306 \\
$8(\mathrm{~Tb})$ & 21.6743 & 2.4083 & 126.2868 & 3.5080 \\
$9(\mathrm{Dy})$ & 21.5659 & 2.3962 & 125.6326 & 3.4898 \\
$10(\mathrm{Ho})$ & 21.6784 & 2.4087 & 126.3003 & 3.5083 \\
$11(\mathrm{Er})$ & 21.3122 & 2.3680 & 124.1938 & 3.4498 \\
$12(\mathrm{Tm})$ & 21.3175 & 2.3686 & 124.2515 & 3.4514 \\
$13(\mathrm{Yb})$ & 21.2450 & 2.3606 & 123.7741 & 3.4382 \\
$14(\mathrm{Lu})$ & 18.6500 & 2.3312 & 96.2735 & 3.4383 \\
$\mathrm{~d}_{\mathrm{La}} / \mathrm{d}_{\mathrm{Lu}}$ & 1.2160 & 1.0809 & 1.3751 & 1.0696 \\
\hline
\end{tabular}

Table S4. Sum of all Ln-O bond lengths, $\Sigma_{\mathrm{Ln}-\mathrm{O}}$, and sum of all $\mathrm{O}-\mathrm{O}$ distances, $\Sigma_{\mathrm{O}-\mathrm{O}}$, obtained from RM1 full geometry optimizations of the LOFs $\left[\mathrm{Ln}_{2}\left(\mathrm{C}_{4} \mathrm{H}_{4} \mathrm{O}_{4}\right)_{3}\left(\mathrm{H}_{2} \mathrm{O}\right)_{2}\right] \cdot \mathrm{H}_{2} \mathrm{O}$, where $\mathrm{Ln}$ is $\mathrm{La}-\mathrm{Lu}$ (except Pm).

\begin{tabular}{ccccc}
\hline f electrons $(\mathrm{Ln})$ & $\Sigma_{\text {Ln-O }}(\AA)$ & Average $(\AA)$ & $\Sigma_{\text {O-O }}(\AA)$ & Average $(\AA)$ \\
\hline $0(\mathrm{La})$ & 23.8867 & 2.6541 & 139.1608 & 3.8656 \\
$1(\mathrm{Ce})$ & 23.8464 & 2.6496 & 138.8246 & 3.8562 \\
$2(\mathrm{Pr})$ & 23.6060 & 2.6229 & 137.2119 & 3.8114 \\
$3(\mathrm{Nd})$ & 22.4758 & 2.4973 & 130.9991 & 3.6389 \\
$4(\mathrm{Pm})$ & -- & --- & -- & -- \\
$5(\mathrm{Sm})$ & 22.7158 & 2.5240 & 132.2981 & 3.6749 \\
$6(\mathrm{Eu})$ & 22.4743 & 2.4971 & 131.0079 & 3.6391 \\
$7(\mathrm{Gd})$ & 22.1495 & 2.4611 & 129.0644 & 3.5851 \\
$8(\mathrm{~Tb})$ & 22.0586 & 2.4510 & 128.6725 & 3.5742 \\
$9(\mathrm{Dy})$ & 22.0452 & 2.4495 & 128.7173 & 3.5755 \\
$10(\mathrm{Ho})$ & 21.9515 & 2.4391 & 127.7609 & 3.5489 \\
$11(\mathrm{Er})$ & 21.8120 & 2.4236 & 127.0525 & 3.5292 \\
$12(\mathrm{Tm})$ & 23.7400 & 2.6378 & 135.8726 & 3.7742 \\
$13(\mathrm{Yb})$ & 21.9690 & 2.4410 & 128.0416 & 3.5567 \\
$14(\mathrm{Lu})$ & 19.1480 & 2.3935 & 97.8243 & 3.4937 \\
$\mathrm{~d}_{\mathrm{La}} / \mathrm{d}_{\mathrm{Lu}}$ & 1.2475 & 1.1089 & 1.4226 & 1.1064 \\
\hline
\end{tabular}

